\title{
SUSTENTABILIDAD AMBIENTAL EN FINCAS CAFETALERAS DESPUÉS DE UN PROCESO DE CERTIFICACIÓN ORGÁNICA EN LA CONVENCIÓN (CUSCO, PERÚ)
}

\section{ENVIRONMENTAL SUSTAINABILITY IN COFFEE FARMS AFTER AN ORGANIC CERTIFICATION PROCESS AT LA CONVENCION (CUSCO, PERU)}

\author{
Fanny Márquez Romero ${ }^{1}$, Alberto Julca Otiniano², Manuel Canto Saenz ${ }^{3}$, Hugo Soplín Villacorta ${ }^{4}$, Silvana \\ Vargas Winstanley ${ }^{5}$ y Pablo Huerta Fernández ${ }^{6}$
}

\begin{abstract}
Resumen
El objetivo de este trabajo fue determinar la sustentabilidad ambiental de las fincas cafetaleras después de un proceso de certificación orgánica en La Convención (Cusco, Perú). Se utilizó el Método de Análisis Multicriterio adaptado para fincas cafetaleras, se consideró que la sustentabilidad se logra cuando el Indicador General Ambiental (IA) es $>2$ puntos, de un máximo de 4 puntos. La población fue de 1520 fincas y se tomó una muestra $(n=61)$. Los responsables de las fincas seleccionadas fueron encuestados en dos momentos, primero cuando trabajaban con un sistema de producción convencional y luego cuando pasaron a trabajar con un sistema certificado de producción orgánica, con un intervalo de tiempo de siete años. Se encontró que el sistema de producción orgánica alcanzó un mayor Indicador General Ambiental (IA $=2.71)$, más alto que el que alcanzó el sistema de producción convencional (IA=2.08). El número de fincas sustentables ambientalmente, aumentó con el proceso de certificación orgánica. Cuando producían café convencional, el $66.60 \%$ de las fincas eran ambientalmente sustentables; la cifra aumentó a 91.10\%, siete años después. El rendimiento de café, aumentó de $665.16 \mathrm{~kg} / \mathrm{ha}$ (cuando se trabajaba con el sistema convencional) a $858.38 \mathrm{~kg} / \mathrm{ha}$ (con el sistema orgánico), es decir hubo un incremento de la productividad de un 29.04\%. La calidad del café, también mejoró y pasó de 80.64 (cuando se trabajaba con el sistema convencional) a 82.56 puntos (con el sistema orgánico). El sistema de producción orgánica alcanzó un mayor Indicador de Sustentabilidad Económica (IK = 2.06), más alto que el que alcanzó el sistema de producción convencional (IK=1.61).
\end{abstract}

Palabras clave: Sustentabilidad ambiental, producción orgánica, agricultura familiar, agroecología, sostenibilidad.

\begin{abstract}
The aim of this study was to define the environmental sustainability of coffee farms after an organic certification process at La Convencion (Cusco, Peru). The Multi Criteria Analysis Method adapted for coffee farms was used; it was considered that sustainability is achieved when the General Environmental Indicator (IA) is $>2$ points, a maximum of 4 points. A sample $(n=61)$ was taken from the population of 1520 farms. Those responsible for the selected farms were surveyed in two stages, first when working with a conventional production system and then when they went to work with a certified organic production system, with a time interval of seven years. It was found out that organic production system achieved a higher General Environmental Indicator $(\mathrm{AI}=2.71)$ than the one reached with conventional production system $(\mathrm{AI}=2.08)$. The number of environmentally sustainable farms increased with organic certification process. $66.6 \%$ of the farms were environmentally sustainable when producing conventional coffee and seven years later, they were $91.10 \%$. The coffee yield increased from $665.16 \mathrm{~kg} /$ ha (with the conventional system) to 858.38 $\mathrm{kg} /$ ha (with the organic system), which means a productivity increase of $29.04 \%$. Coffee quality also improved from 80.64 points (with the conventional system) to 82.56 points (with the organic system). The organic production system achieved a higher Indicator of Economic Sustainability (IK $=2.06)$, than the one reached with the conventional production system $(\mathrm{IK}=1.61)$.
\end{abstract}

Key words: Environmental sustainability, organic production, family farming, agro-ecology, sustainability. 


\section{Introducción.}

En el Perú, el café es el principal producto de exportación agrícola seguido de los espárragos y representa cerca de la mitad de las exportaciones agropecuarias. Según el Instituto Nacional de Estadística e Informática del Perú (INEI) en su informe sobre el censo Agropecuario del 2013, señala que se destinaron 425 mil hectáreas a la producción del café en el 2012 y la Junta Nacional del Café de Perú (JNC) en el 2013 indica que están distribuidas en 338 distritos de 16 regiones del Perú, siendo el sustento económico directo de más de 150 mil familias y genera aproximadamente 2 millones de puestos de trabajo directos e indirectos en la cadena productiva.

La provincia de La Convención en Cusco es la segunda provincia más extensa del Perú y cuenta con una extensión de $30061.82 \mathrm{~km}^{2}$ de territorio (Encinas, 2007), donde se hallan tres regiones: Sierra, Ceja de Selva y Selva. El principal producto agrícola es el café (INEI, 2013), producido bajo sombra en sistemas asociados a especies forestales que pertenecen a los géneros: Inga (Guabas), Albizzia sp. (Albizzia) y Leucaena sp. (Chamba) de manera simultánea, o sea que los componentes agrícolas y arbóreos se encuentran en el mismo terreno durante toda la duración del sistema, en estos sistemas se puede encontrar especies forestales de alturas de 20 a 35 metros, en forma muy aleatoria como: Cedrela odorata (Cedro), Juglans olanchana (Nogal), Calophyllum brasiliense, Swietenia sp. (Caoba) y Cordia alliodora (Laurel) (Márquez, 2015). Dentro de los cafetales se encuentran también especies frutales como Musa spp. (Plátanos), Citrus spp. (Cítricos), Persea americana (palto), Mangifera indica (Mango) y Annona muricata (Guanábana) formando un ecosistema diverso tipo vergel, al respecto Merma (2011), menciona que este tipo de agroecosistemas produce bajos rendimientos de café pergamino seco (menores de $506 \mathrm{~kg} / \mathrm{ha}$ ) debido a la baja densidad de plantas de café pero con alta diversidad biológica que favorece el mantenimiento del paisaje y la seguridad alimentaria. Según Swift et al., (2004), la biodiversidad es importante para la regulación del sistema ya que, entre otras funciones, proporciona hábitats y nichos ecológicos para los enemigos naturales y es la base de la diversidad heterotrófica.

La caficultura en la provincia de La Convención en Cusco se inició antes del año 1846 (Aparicio, 1999), produciéndose café de alta calidad pero bajo sistemas tradicionales andinos que hoy son considerados como inadecuados, sin embargo, actualmente se continúa con las prácticas poco conservativas (Merma, 2011) denominado como sistema tradicional; de forma paralela se incentiva los sistemas orgánicos desde 1996 a través de la Central de Cooperativas COCLA y sus cooperativas socias (Márquez, 2004), sistema que fue mejorando en forma paulatina y actualmente se practica con la adopción de técnicas agroecológicas como la no utilización de agroquímicos, trazabilidad del producto y registro de actividades, se induce el incremento del rendimiento mediante la aplicación de podas, reposición de nutrientes y conservación de suelos mediante la utilización de barreras vivas y muertas, curvas a nivel, cobertura muerta, manejo de la biodiversidad conservando y recuperando bosques, manejo ecológico de plagas mediante el control biológico y etológico y adecuada cosecha y post cosecha que garanticen un café de alta calidad (Márquez, 2015).

Los sellos más difundidos para café tienen un énfasis ambiental claramente marcado y el sello orgánico prohíbe el uso de insumos industriales (Guhl, 2009; Altieri, 1999), esto se traduce en una menor productividad del cafetal, esta reducción productiva se compensa con el sobreprecio que pagan los consumidores (Gobbi, 2000). El cultivo del café orgánico peruano es desarrollado por diversas cooperativas y empresas privadas, quienes operan bajo las normas internacionales de JAS (Japanese Agricultural Standards), USDA NOP (The National Organic Program standards of the United States Department of Agriculture) y UE = EU 834/2007 - EU 889/2008 para el mercado Europeo, financiado con recursos propios o con apoyo internacional y entre los criterios de certificación destaca la prohibición del uso de agroquímicos y el incremento del porcentaje y diversidad de sombra como una actividad para disminuir el impacto del cambio climático que se presenta con temperaturas máximas diarias superiores a $30^{\circ} \mathrm{C}$ y precipitaciones pluviales cada vez más reducidas, así como para conservar la fertilidad de los suelos (Márquez, 2015) mediante la mineralización de la cobertura de los suelos. En la zona de estudio, los sistemas producción de café convencional practican una agricultura tradicional y con bajo uso de insumos externos (Márquez, 2015 y Merma, 2011).

La sustentabilidad ambiental evaluada en este trabajo se basa en el método que es capaz de realizar comparaciones entre agroecosistemas y monitorea los avances a lo largo del tiempo hacia una mayor o menor sustentabilidad en el proceso de transición, aplicando enfoque cualitativo bajo la utilización de indicadores propuesto por Sarandón y Flores (2009).

La sustentabilidad ecológica significa el mantenimiento del capital natural, es decir, vivir dentro de la capacidad productiva del planeta (Onaindia, 2007), debido a que la humanidad depende de la diversidad biológica y del flujo de servicios de los ecosistemas, que son los beneficios que la sociedad puede obtener de los ecosistemas. Determinar el nivel de sustentabilidad ambiental o ecológica de los agroecosistemas cafetaleros nos permite conocer el efecto que causó la implementación de las normas de producción orgánica y su impacto en la conservación de los ecosistemas cafetaleros de La Convención. 


\section{Materiales y métodos.}

La investigación se realizó en la provincia de $\mathrm{La}$ Convención de la Región Cusco en Perú, Ubicada entre las latitudes sur $11^{\circ} 15^{\prime}$ y $13^{\circ} 30^{\prime}$ y los meridianos $72^{\circ}$ y $74^{\circ}$ Longitud Oeste. El clima según la clasificación de Köppen es Tropical (Aw), la temperatura media máxima anual es de $29{ }^{\circ} \mathrm{C}$ mientras que la media mínima anual es de $18{ }^{\circ} \mathrm{C}$; las precipitaciones anuales en promedio no superan los $1500 \mathrm{~mm}$, con valores de 900 a 3000mm de rango anual y suceden con mayor frecuencia entre diciembre y marzo presentando una marcada estación seca durante los meses de mayo a setiembre. La humedad relativa varía de 65 a $90 \%$ con un promedio anual de 77\%.Los suelos corresponden al orden de los ultisoles, oxisoles y fluvisoles con presencia de óxido de hierro, textura franco-arcilloso, ácidos y de baja a mediana fertilidad natural (GRC, 2005).

\section{Tamaño y selección de la muestra}

La población de unidades agropecuarias (UA) cafetaleras orgánicas en la zona de estudio fue de 1520 UA, de ésta se tomó una muestra (n=61). El tamaño de muestra se calculó usando el método de Murray \& Larry (2009). En la Tabla 1 se presenta la distribución de la muestra en cada uno de los distritos estudiados, los agricultores se seleccionaron de forma aleatoria entre la población disponible en el marco muestral de cada distrito.

Tabla 1. Tamaño de muestra por distritos en la zona de estudio.

\begin{tabular}{llclr}
\hline Distritos & \multicolumn{2}{l}{$\begin{array}{l}\mathbf{N}^{\circ} \\
\text { cafetaleros orgánicos }\end{array}$} & $\begin{array}{c}\text { Agricultores } \\
\text { \% }\end{array}$ & Muestra \\
\hline Quellouno & $\mathrm{N}_{1}$ & 349 & 0.23 & 14 \\
Echarate & $\mathrm{N}_{2}$ & 548 & 0.36 & 22 \\
Vilcabamba & $\mathrm{N}_{3}$ & 149 & 0.10 & 6 \\
Santa Ana & $\mathrm{N}_{4}$ & 249 & 0.16 & 10 \\
Santa Teresa & $\mathrm{N}_{5}$ & 100 & 0.07 & 4 \\
Maranura & $\mathrm{N}_{6}$ & 125 & 0.08 & 5 \\
TOTAL & $\mathbf{N}$ & $\mathbf{1 5 2 0}$ & 1 & $\mathbf{6 1}$ \\
\hline
\end{tabular}

N= Población

\section{Construcción de indicadores}

Los indicadores se construyeron de acuerdo a la metodología y el marco conceptual propuesto por Sarandón (2002) y Sarandón \& Flores (2009), siguiendo los lineamientos de Smyth \& Dumansky (1995) y Astier et al. (2008) considerando que mediante este método se mide la sustentabilidad de forma comparativa entre agroecosistemas y facilita el monitoreo delos avances a lo largo del tiempo hacia una mayor o menor sustentabilidad en el proceso de transición, aplicando el enfoque cualitativo. Se consideró al indicador como una variable, seleccionada y cuantificada que hace clara una tendencia que de otra forma no es fácilmente detectable (Sarandón, 2002). Se eligieron indicadores que fueran fáciles de obtener, de interpretar, que brindaran la información necesaria y que permitieron detectar las tendencias en el ámbito de las muestras seleccionadas. Estos estuvieron compuestos a su vez por subindicadores que fueron cuantificados.

Estandarización y ponderación de los indicadores

Para permitir la comparación de las fincas y facilitar el análisis de la dimensión ambiental de la sustentabilidad, los datos fueron estandarizados, mediante su transformación a una escala, para cada indicador, de 0 a 4, siendo 4 el mayor valor de sustentabilidad y 0 el más bajo. Todos los valores, independientemente de su unidad original, se transformaron o adecuaron a esta escala. Esto posibilitó la integración de varios indicadores de distinta naturaleza, en otros más sintéticos o robustos. Posteriormente, los indicadores fueron ponderados multiplicando el valor de la escala por un coeficiente de acuerdo a la importancia relativa de cada variable respecto a la sustentabilidad. Este coeficiente multiplica, tanto el valor de las variables que forman el indicador, como el de los indicadores, para construir indicadores de mayor nivel o índices. La ponderación, es un paso inevitable, que puede hacerse por consenso, por medio de la consulta con expertos en el tema (Gayoso \& Iroume, 1991 y Astirraga, 2003), o teniendo en cuenta la opinión de los propios agricultores (Roming et al., 1996; Lefroy et al., 2000). En este trabajo, la ponderación se realizó por discusión y consenso entre técnicos, expertos y agricultores líderes que participaron en la evaluación. El peso de cada indicador refleja la importancia del mismo en la sustentabilidad.

$\underline{\text { Indicadores ambientales seleccionados }}$

A. Conservación de la biodiversidad de suelo.

Un sistema es sustentable si las prácticas mantienen o mejoran la biodiversidad en el suelo. Para construir este indicador se tuvieron en cuenta dos subindicadores:

$\mathrm{A}_{1}$ - Manejo de la cobertura vegetal. La misma provee al suelo de una protección contra las radiaciones solares altas y prolongadas y temperaturas mayores a $30{ }^{\circ} \mathrm{C}$, así como disminuye el riesgo de erosión. (4): 100\% de cobertura; (3): 99 a 75 \%; (2): 74 a $50 \%$; (1): 49 a $25 \%$; (0): $<25 \%$ de cobertura.

$\mathrm{A}_{2^{-}}$Diversificación de cultivos. (4): Establecimiento totalmente diversificado, con asociaciones de cultivos; (3): Alta diversificación de cultivos, con asociación media entre ellos; (2): Diversificación media, con muy bajo nivel de asociación entre ellos; (1): Poca diversificación de cultivos, sin asociaciones; (0): Monocultivo.

B. Riesgo de erosión.

Un sistema es sustentable si logra minimizar o evitar la pérdida de suelo debido a la erosión (en este caso, hídrica). Se tuvieron en cuenta 3 subindicadores:

$\mathrm{B}_{1}$ - Pendiente o grado de inclinación del área predominante de los cultivos. (4): del 0 al $5 \%$; (3): del 6 al 15 \%; (2): del 16 al 30 \%; (1): del 31 al 45 \%; (0): mayor al $45 \%$ 
$\mathrm{B}_{2}$ - Cobertura vegetal sobre suelo. La misma le provee al suelo una protección contra temperaturas superiores a $30^{\circ} \mathrm{C}$ y disminuye la velocidad de la escorrentía superficial. (4): 100\% de cobertura; (3): 75 a $99 \%$; (2): 74 a $50 \%$; (1): 25 a $49 \%$; (0): de 0 a $24 \%$ de cobertura.

$\mathrm{B}_{3}$ - Conservación de suelos. El sistema sustentable es aquel que conserva la cantidad y calidad de sus suelos. Variable: Labores o técnicas realizadas por el productor con respecto a la conservación de suelos en áreas mayores al 50\% de cultivo de café y de los principales cultivos de renta complementarios. (4): Curvas de nivel o terrazas; (3): Barreras vivas y muertas; (2): Barreras muertas; (1): Surcos en tresbolillo (las plantas en una siembra se ponen en filas paralelas, de modo que cada planta de una fila quede frente al medio de dos plantas de la fila siguiente) orientados a la pendiente; (0): Surcos paralelos a la pendiente sin ninguna barrera.

C. Manejo de la Biodiversidad.

La biodiversidad presente en los ecosistemas agrícolas proporciona el alimento y los medios para producirlo. La diversidad de plantas y animales que consumimos son componentes de la diversidad agrícola que podemos apreciar a simple vista. Igualmente importantes, aunque menos visibles, son los miles de organismos presentes en el suelo, los polinizadores y los enemigos naturales de las plagas y enfermedades, cuya función reguladora constituye el soporte de la producción agrícola. El efecto del sistema de manejo de la finca sobre la biodiversidad, se evaluó a través de los siguientes dos subindicadores:

$\mathrm{C}_{1}$ - Biodiversidad vegetal. Variable: Diversidad de especies forestales, de cobertura, arvenses y de autoconsumo. (4): Establecimiento totalmente diversificado, con asociaciones entre ellos y con bastante vegetación natural que cubre los suelos; (3): Alta diversificación de especies, con asociación media entre ellos, alta presencia de hierbas benéficas sobre los suelos; (2): Diversificación media, con muy bajo nivel de asociación entre ellos, escasa diversidad de hierbas sobre suelos; (1): Poca diversificación de especies, sin asociaciones, presencia de dos o tres hierbas sobre suelos; (0): Sin diversidad en especies de sombra y presencia de malezas de efecto negativo sobre suelos.

$\mathrm{C}_{2}$ - Área de zonas de conservación. Las zonas de conservación incluyen bosques, pastizales, pantanos, orillas de ríos y riachuelos, zonas de amortiguamiento, donde no se realice labores agrícolas y por el contrario estén adecuadamente delimitadas y conservadas. (4): mayor de 2.1 ha; (3): de 1.1 a 2.00 ha; (2): de 0.51 a 1.00ha; (1): de 0.1 a 0.5 ha; (0): No tiene ningún área de conservación.

El indicador que mide el grado de cumplimiento de la dimensión ambiental (IA), obtiene como valor máximo 4 y se valora como sustentable cuando alcanza o supera el puntaje de 2 y se calculó de la siguiente manera:
Indicador General Ambiental (IA)

$=\frac{\left(A_{1}+A_{2}\right) / 2+\left(B_{1}+B_{2}+B_{3}\right) / 3+\left(C_{1}+C_{2}\right) / 2}{3}$

Los productores de café orgánico seleccionados fueron encuestados para obtener la información relacionada con la sustentabilidad ambiental (después del proceso de certificación). La información (antes del proceso de certificación) se obtuvo de la línea base preparada por las organizaciones responsables, es decir cuando eran productores de café convencional, mediante encuestas y visitas a las fincas. El periodo entre el “antes” y “después” fue de siete años; periodo considerado suficiente para ver los cambios de un sistema de producción de café convencional a un orgánico, por lo que debemos resaltar que son las mismas unidades evaluadas en dos tiempos diferentes.

\section{Resultados y discusión.}

En la Tabla 2 se observa que el sistema de producción orgánica alcanzó 2.71 puntos como Indicador General Ambiental (IA), cifra mayor al 2.08 que tenía cuando se trabajaba como un sistema convencional. Un IA $>2$, permite considerar a ambos sistemas de producción como ambientalmente sustentables (Sarandón et al., 2006). Pero, es importante señalar que en el sistema convencional, el subindicador riesgo de erosión (RE) es menor a 2, por lo tanto no logra el valor mínimo de sustentabilidad debido principalmente al bajo porcentaje de cobertura de suelos que no alcanza el 50\%. Esta falta de cobertura, incrementa el riesgo de erosión en zonas de pendiente pronunciada como ocurre en los cafetales por lo que dificulta la conservación de suelos. Lo contrario ocurre en el sistema de producción orgánico, se tiene una mayor área cubierta con especies vivas instaladas o con cobertura muerta, por lo tanto el riesgo de erosión hídrica disminuye, además de impedir el crecimiento delas arvenses en los cafetales. Puertas (2010), indica que las coberturas vegetales reducen la necesidad de mano de obra para el manejo y control de arvenses. Este y otros tipos de coberturas pueden tener otros efectos positivos sobre el suelo, por ejemplo, Erenstein (2003), señala que el mulch incrementa la disponibilidad de agua en el suelo debido a que disminuye la velocidad y energía de la escorrentía y aumenta la infiltración. Además, en este estudio, la diversidad vegetal (C1) aumentó de 2.44 a 3.61 del sistema convencional a orgánico, debido al incremento de la diversidad de especies de sombra y la cobertura vegetal, factores que disminuyen los efectos negativos que podrían tener, sobre el cultivo de café, la estacionalidad de las lluvias, temperaturas superiores a $28^{\circ} \mathrm{C}$, radiación solar intensa y prolongada y la baja fertilidad de los suelos. Esta mayor diversidad vegetal, permite no sólo obtener leña, sino también frutos que favorece la mayor diversidad 
Tabla 2. Sustentabilidad ambiental del sistema cafetalero orgánico y convencional en La Convención, Cusco.

\begin{tabular}{|c|c|c|c|c|c|c|c|c|c|c|c|}
\hline \multirow{2}{*}{$\begin{array}{l}\text { Indicadores } \\
\text { Ambientales }\end{array}$} & \multicolumn{3}{|c|}{$\begin{array}{c}\text { Conservación de la vida de } \\
\text { suelo (A) } \\
\end{array}$} & \multicolumn{4}{|c|}{ Riesgo de erosión (B) } & \multicolumn{3}{|c|}{$\begin{array}{l}\text { Manejo de la Biodiversidad } \\
\text { (C) }\end{array}$} & \multirow{2}{*}{$\begin{array}{c}\text { IA } \\
\text { Indicador } \\
\text { General } \\
\text { Ambiental }\end{array}$} \\
\hline & MCob. & Div. & VS & Pend. & Cob. & ConS & $\mathrm{RE}$ & Biodiv & Acons & MB & \\
\hline Sistema & $\left(\mathrm{A}_{1}\right)$ & $\left(\mathrm{A}_{2}\right)$ & $\left(\mathrm{A}_{1}+\mathrm{A}_{2}\right) / 2$ & $\left(\mathrm{~B}_{1}\right)$ & $\left(\mathrm{B}_{2}\right)$ & $\left(\mathrm{B}_{3}\right)$ & $\left(\mathrm{B}_{1}+\mathrm{B}_{2}+\mathrm{B}_{3}\right) / 3$ & $\left(\mathrm{C}_{1}\right)$ & $\left(\mathrm{C}_{2}\right)$ & $\left(\mathrm{C}_{1}+\mathrm{C}_{2}\right) / 2$ & $(\mathrm{~A}+\mathrm{B}+\mathrm{C}) / 3$ \\
\hline $\begin{array}{l}\text { Convencional } \\
\text { (antes) } \\
\text { Orgánico }\end{array}$ & 1.87 & 2.44 & 2.16 & 0.79 & 1.87 & 2.26 & 1.64 & 2.44 & 2.44 & 2.44 & $2.08 * *$ \\
\hline (después) & 2.26 & 3.61 & 2.93 & 0.79 & 2.26 & 3.25 & 2.10 & 3.61 & 2.57 & 3.09 & $2.71 * *$ \\
\hline
\end{tabular}

de animales silvestres en un sistema de producción orgánica.

Los datos son la media de 61 muestras evaluadas en dos tiempos.

MCob.= Manejo de la cobertura vegetal; Div.= Diversidad de cultivos; VS = Conservación de la biodiversidad del suelo; Pend.= Pendiente predominante; $\mathrm{Cob}=$ Cobertura, ConS. $=$ Conservación de suelos; RE = Subindicador de Riesgo de erosión; Biodiv. $=$ Biodiversidad vegetal; Acons. $=$ Área de zonas de conservación; $\mathrm{MB}=$ Manejo de la biodiversidad; IA = Indicador general ambiental** Sustentable, por tener valor $>2$.

Otros estudios para evaluar sustentabilidad ambiental han sido realizados en los últimos años. En el Cusco (Perú), se reportó la sustentabilidad ecológica en sistemas de producción agrícola que tenían como cultivo predominante al café (IA=2.40), cacao $(\mathrm{IA}=2.83)$, té $(\mathrm{IA}=2,47)$, plátano ( $\mathrm{IA}=2.53)$, cítricos $(\mathrm{IA}=2.30)$, papaya $(\mathrm{IA}=2.10)$ y mango $(\mathrm{IA}=3.10)$; mientras que cuando el cultivo predominante fue coca, el IA fue de 1.50, es decir ecológicamente no fue sustentable (Merma, 2011). En Manabí (Ecuador), al evaluar las fincas cafetaleras, se encontró que el 95\% de estas fueron ecológicamente sustentables y un 5\% no lo fueron (Santistevan, 2013).

En Espíritu Santo (Brasil), en el cultivo de café, se usaron indicadores estratégicos para evaluar los sistemas de producción usando las llamadas Buenas Prácticas Agrícolas (BPA), sistema de producción orgánico y sistema de producción convencional. Se encontró que el sistema BPA es considerado como alternativo y de mayor aporte tecnológico y presentó una tendencia de mejora creciente para los indicadores de sustentabilidad; mientras que el sistema orgánico necesita tener continuidad en los procesos de transición, se debe continuar con el rediseño del agroecosistema y el uso de tecnologías más apropiadas (De Muner, 2011).

En Dagua, Valle del Cauca (Colombia), mediante el Índice de Sustentabilidad de Sistemas Productivos Agrícolas (ISSPA) se evaluó la integridad agroecológica de predios, considerando factores como manejo de suelos y coberturas, de agua, de residuos sólidos, aspectos socio-económicos y políticoinstitucionales, entre otros. Se encontró que el 93\% de

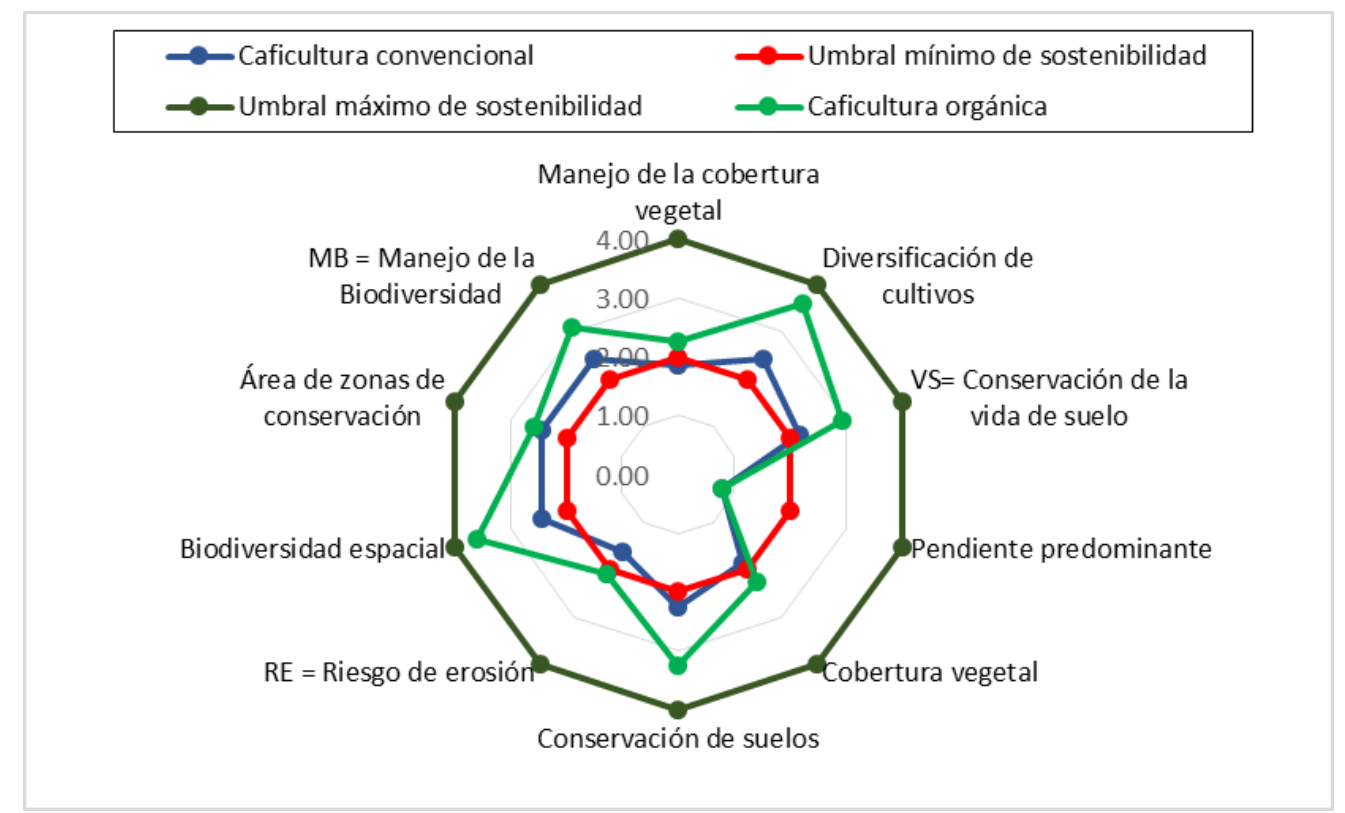

Figura 1. Comparativo de los componentes del Indicador Ambiental (IA) en agroecosistemas de café orgánico y convencional en la provincia de La Convención-Cusco. 
los predios superó el umbral de integridad ecológica, es decir, poseen sistemas productivos agrícolas sustentables (Cerón et al., 2014).

En la Figura 1, se observa que el sistema de producción orgánica mantiene alto riesgo de erosión debido a las pendientes pronunciadas en las que se instaló el café, aspecto que no puede cambiar, pero puede ser mitigado mediante el incremento de cobertura y conservación de suelos.

La diversificación de cultivos, biodiversidad espacial y cobertura vegetal en el sistema orgánico incorporan a las especies de renta alternativa, de sombra, de autoabastecimiento y forestales y son subindicadores que obtuvieron mayores valores respecto al sistema convencional. Debido al mantenimiento de la diversidad biológica y protección de los suelos como lo encontrado por Farfán (2007) y Muschler (2001) se logra disminuir la evapotranspiración, fijar nitrógeno en los suelos y además ofrecen servicios ambientales no sólo al cultivo sino al paisaje en su conjunto, tales como secuestro de carbono, mantenimiento de diversidad biológica y protección de suelos.

El indicador Conservación de la vida del suelo presenta un aumento de 2.16 a 2.93 de convencional a orgánico, relacionado con la mayor presencia de micro y macrofauna por el incremento de la cobertura del suelo con mulch, al respecto Márquez (2015) indica que también incrementa la cantidad de bacterias y hongos y Bartz, et al.,(2009) confirma que la biomasa, densidad de población y diversidad de especies de lombrices son indicadores sensibles de las prácticas de manejo orgánico y que están influenciadas por el tipo de suelos.

Los subindicadores, Área de zonas de conservación y Manejo de la cobertura vegetal, son puntos críticos porque no han mejorado de lo convencional a orgánico, sobre las causas, Márquez (2015), menciona que es efecto del minifundio con unidades agrícolas de menos de 5 hectáreas, alto porcentaje de fincas con pendientes pronunciadas, alto costo de producción, baja disponibilidad de mano de obra y precio del café pergamino seco dependiente de las bolsas de valores, aspectos que están poniendo en riesgo la sostenibilidad de las fincas así mismo Cerón et al., (2014) indican que el "manejo de suelos y coberturas" constituye el factor que atenta contra la integridad ecológica de los sistemas productivos agrícolas en comparación de "residuos sólidos", "aspectos socioeconómicos" y "político-institucionales" que favorecieron la sostenibilidad agroecológica de las fincas evaluadas.

La Figura 2, muestra que relación porcentual de las fincas que lograron sustentabilidad en los subindicadores ambientales evaluados, encontrándose que a nivel convencional las fincas sustentables en conservación de la vida del suelo, riesgo de erosión y manejo de la biodiversidad fueron el 68.8, $37.7 \mathrm{y}$ $70.49 \%$ respectivamente a diferencia del orgánico donde el 100, 72.13 y $91.80 \%$ lograron ser sustentables en las mismas variables. Estos resultados reflejan que a pesar de tener un indicador ambiental general sustentable el porcentaje de fincas convencionales que no lograron sustentabilidad fue de $39.33 \%$, cifra mayor al $8.20 \%$ de las fincas orgánicas.

Para que una finca sea considerada sustentable las fincas deben haber obtenido valores mayores a 2 en todos los indicadores. Con este criterio, cuando las fincas trabajaban con el sistema convencional, 37 fincas (60.66\%) eran ambientalmente sustentables, esta cifra aumentó a 56 (91.8\%) cuando eran fincas productoras de café orgánico (Figura 3).

En el mismo estudio, se realizó una evaluación de la sustentabilidad económica, encontrándose que el sistema de producción orgánica alcanzó un mayor Indicador de Sustentabilidad Económica (IK = 2.06), más alto que el que alcanzó el sistema de producción convencional (IK=1.61). Estos resultados se explican por diversos factores, pero especialmente por el rendimiento de café, que aumentó de $665.16 \mathrm{~kg} / \mathrm{ha}$ (cuando se trabajaba con el sistema convencional) a $858.38 \mathrm{~kg} / \mathrm{ha}$ (con el sistema orgánico), es decir hubo un incremento de la productividad de un $29.04 \%$. La calidad del café, también mejoró y pasó de 80.64 (cuando se trabajaba con el sistema convencional) a 82.56 puntos (con el sistema orgánico). Pero en ambos sistemas de producción el café fue catalogado como "muy buenos" porque el rango para este tipo de café es de 80 a 89 puntos; sin llegar a recibir el calificativo de "sobresalientes" porque para esa categoría el rango es de 90 a 100 puntos, según la Asociación Americana de

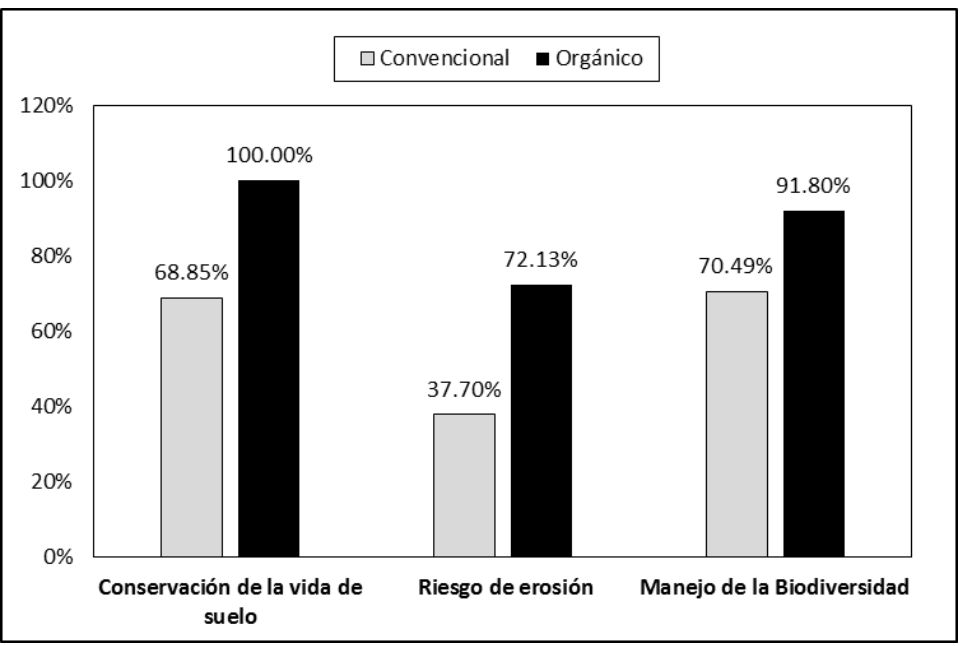

Figura 2. Nivel de Conservación de la vida de suelo, Riesgo de erosión y manejo de la biodiversidad en fincas de café convencional y orgánico en La Convención-Cusco. 


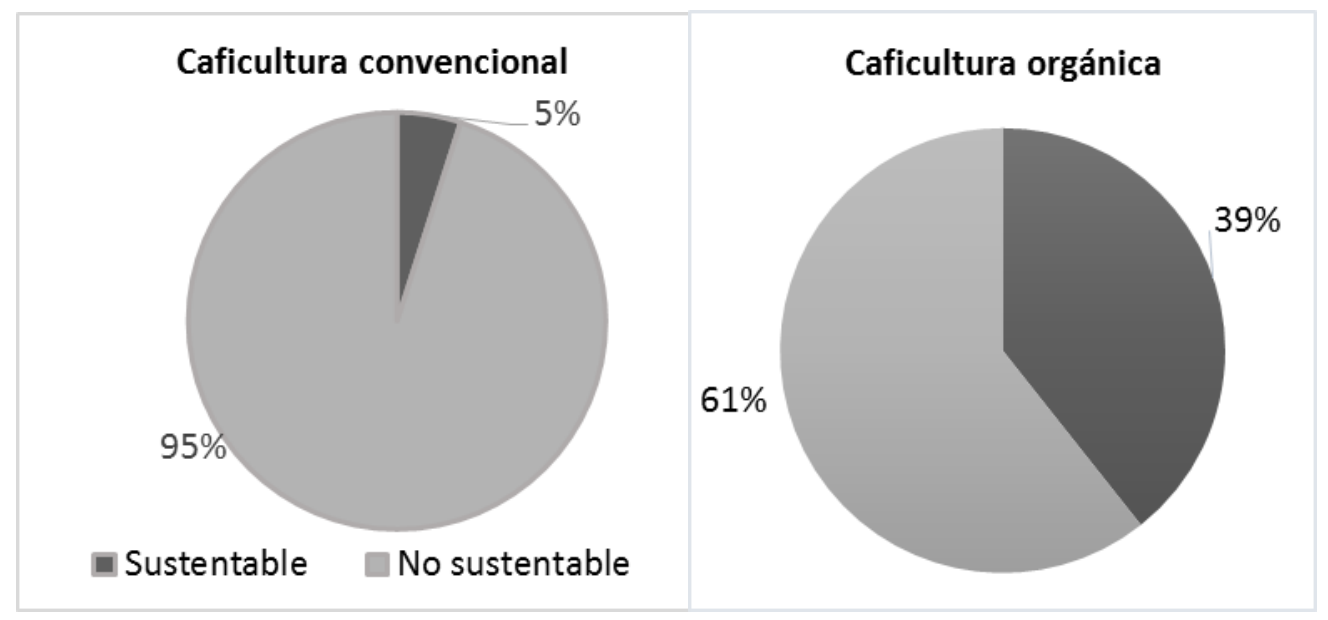

Figura 3. Porcentaje de fincas cafetaleras ambientalmente sustentables, antes del proceso de certificación (convencional) y después (orgánica) en La Convención, Cusco.

Cafés Especiales (SCAA, 2008). Estos resultados muestran que el sistema de producción orgánica, permitiría un mayor ingreso neto mensual para el caficultor y el sistema puede ser considerado económicamente sustentable.

\section{Conclusiones.}

- El sistema de producción orgánica alcanzó un mayor Indicador General Ambiental (IA = 2.71), más alto que el obtenido por el sistema de producción convencional (IA=2.08).

- El número de fincas sustentables ambientalmente, aumentó con el proceso de certificación orgánica. Cuando producían café convencional, el 66.60\% de las fincas eran ambientalmente sustentables; la cifra aumentó a $91.10 \%$, siete años después.

- El rendimiento de café, aumentó de 665.16 kg/ha (cuando se trabajaba con el sistema convencional) a $858.38 \mathrm{~kg} / \mathrm{ha}$ (con el sistema orgánico), es decir hubo un incremento de la productividad de un 29.04\%.

- La calidad del café también mejoró y pasó de 80.64 (cuando se trabajaba con el sistema convencional) a 82.56 puntos (con el sistema orgánico).

- El sistema de producción orgánica obtuvo un Indicador de Sustentabilidad Económica (IK = 2.06), más alto que el que alcanzó el sistema de producción convencional (IK=1.61).

\section{Agradecimientos.}

A la Central COCLA, BIO AZUL SAC, CAPACY y a todos los productores de café orgánico de La Convención, por su perseverancia y dedicación en el cultivo de café.

\section{Literatura citada.}

Altieri M.A. 1999. Agroecología, Bases científicas para una agricultura sustentable. Editorial Nordan-Comunidad. Montevideo. 325 pp.
Aparicio V.M. 1999. De Vilcabamba a Camisea. Historiografía de la provincia de La Convención. Cusco, Ediciones especiales UNSAAC- Siglo XX. 551 pp.

Astier M, Masera O.R. \& Galván-Miyoshi Y. 2008. Evaluación de sustentabilidad. Un enfoque dinámico y multidimensional. Valencia, España.

Astigarraga, E. 2003. El método delphi. San Sebastián, Spain: Universidad de Deusto.

Bartz M., Brown G., Pasini A., Oliveira Fernandes J., Curmi P., Dorioz J. \& Ralisch R. 2009. Comunidades de Minhocas em cultivo de café orgânico e convencional. Pesq. agropec. bras.Vol.44 no.8. Brasília. Brasil.

Cerón W. L., Escobar Y. C. \& Díaz Á. J. Á. 2014. Evaluación agroecológica de los sistemas productivos agrícolas en la microcuenca Centella (Dagua, Colombia). Colombia Forestal, 17(2), 161-179.

De Muner L.H. 2011. Sostenibilidad de la Caficultura Arábica en el Ámbito de la Agricultura Familiar en el Estado de Espírito Santo - Brasil. Tesis del Programa de Doctorado en Recursos Naturales y Sostenibilidad. Línea de Investigación Agroecología. Instituto de Sociología y Estudios Campesinos. Departamento de Ciencias Sociales y Humanidades. Universidad de Córdoba. España. 259 pp.

Encinas A.M. 2007. Historia de la provincia de La Convención. Tomo I. Siglos XVI - XIX. Centro Cultural José Pío Aza. Misioneros Dominicos y Centro Bartolomé de las Casas. Perú. 325pp.

Erenstein O. 2003. Smallholder conservation farming in the tropics and subtropics: A guide to the development and dissemination of mulching with crop residues and cover crops. Agriculture, Ecosystems and Environment 100(1):17-37.

Farfán F. 2007. Producción de café en Sistemas agroforestales. En Sistemas de producción de café en Colombia. Cap. VIII. Chinchiná, Cenicafé. 309 pp.

Gayoso, J. \& Iroumé, A. 1991. Metodología para estimar la fragilidad de terrenos forestales. Medio Ambiente 11(2): 13-24.

Gobbi J.A. 2000. Is Biodiversity-friendly Coffee Financially Viable? An Analysis of Five Different Coffee Production 
Systems in Western El Salvador. Ecological Economics 33: 267-281.

GRC (GOBIERNO REGIONAL DEL CUSCO). 2005. Zonificación Económica y Ecológica del Cusco. ZEECusco. Instituto de Manejo de Agua y Medio Ambiente Dirección de Estudios y Proyectos de Gestión Ambiental. Cusco-Perú. 110 pp.

Guhl A. 2009. Café, bosques y certificación agrícola en Aratoca, Santander. Revista de Estudios Sociales, 32: 114-125.

INEI (Instituto Nacional de Estadística e Informática). 2013. Resultados Definitivos del IV Censo Nacional Agropecuario - 2012. Perú. Consulta en Línea.

JNC (Junta Nacional del Café). 2013. Estadísticas de producción y comercialización mundial. Consulta en línea. Perú.

Lefroy R.D.B., Bechstedt H.D. \& Rais M. 2000. Indicators for sustainable land management based on farmer survey in Vietnam, Indonesia and Thailand. Agriculture, Ecosystem and Environment, 81(1):137-146.

Márquez R.F. 2004. Diagnóstico y propuesta de un sistema de gestión ambiental en la Central de Cooperativas Agrarias COCLA. Tesis de Maestría en Ciencias Ambientales. Escuela de Post Grado de la Universidad Nacional Agraria La Molina. Lima-Perú.

Márquez R.F. 2015. Sustentabilidad de la caficultura orgánica en La Convención-Cusco. Tesis Doctoral en Agricultura Sustentable. Escuela de Post Grado de la Universidad Nacional Agraria La Molina. Lima-Perú.

Merma M.I. 2011. Evaluación y diseño de fincas en selva alta bajo sistemas de cultivos prevalentes en La ConvenciónCusco. Tesis Doctoral en Agricultura Sustentable. Escuela de Post Grado de la Universidad Nacional Agraria La Molina. Lima-Perú.

Murray R. S. \& Larry J. S. 2009. Estadística. 4ta edición. Mc Graw-Hill. México D.F. México.

Muschler R.G. 2001. Shade improves coffee quality in a suboptimal coffee zone of Costa Rica. Agroforestry Systems (85): 131-139.

Onaindia O.M. 2007. Sostenibilidad ecológica. Forum de sostenibilidad. Cátedra UNESCO sobre Desarrollo
Sostenible y Educación Ambiental Universidad del PaísVasco/Euskal Herriko Unibertsitatea. Campus de Leioa. 1:39-49.

Puertas R.F. 2010. Índices de Calidad del suelo y parámetros de Crecimiento de Cultivos de cobertura en una Plantación de Cacao (Theobroma cacao L.). Tesis Doctoral en Agricultura Sustentable de la Universidad Nacional Agraria La Molina. 125pp.

Roming D.E., Garlynd M. J. \& Harris R. F. 1996. Farmerbased assessment of soil quality: a soil health scorecard. Pp. 39-60. In: J. W. Doran and A. J. Jones (Eds.). Methods for assessing soil quality. SSSA Special Publication No. 49. SSSA, Madison.

Santisteban M.M. 2013. Sustentabilidad de las Fincas Cafetaleras en Jipijapa Manabí, Ecuador. Tesis de Maestría en Agricultura Sustentable, Universidad Nacional Agraria La Molina. UNALM. Perú.

Sarandón S.J. \& Flores C.C. 2009. Evaluación de la sustentabilidad en agroecosistemas: Una propuesta metodológica. Agroecología 4: 19-28.

Sarandón S.J. 2002. El desarrollo y uso de indicadores para evaluar la sustentabilidad de los agroecosistemas. En Agroecología. El camino hacia una agricultura sustentable (Sarandón SJ, ed.). Ediciones Científicas Americanas, Capítulo 20: 393-414.

Sarandón S.J., Zuluaga M.S., Cieza R., Gómez C., Janjetic L. \& Negrete E. 2006. Evaluación de la sustentabilidad de sistemas agrícolas de fincas en Misiones, Argentina, mediante el uso de indicadores. Revista Agroecología 1: 19-28.

SCAA. 2008. Protocolo para análisis sensorial de café. Metodología SCAA cupping protocols TSC-SCAA Rev. December 2008 Doc V.

Smyth A.J. \& Dumansky, J. 1995. A framework for evaluating sustainable land management. Canadian Journal of Soil Science 75: $401-406$.

Swift M.J., Izac M.N. \& Van Noordwijk M. 2004. Biodiversity and ecosystem services in agricultural landscapes- are we asking the right questions? Agriculture, Ecosystems and Environment 104: 113-134.

\footnotetext{
${ }^{1}$ Universidad Nacional Agraria La Molina. Programa Doctorado Agricultura Sustentable. Facultad de Agronomía. e-mail: famarquez222@yahoo.com

2 Universidad Nacional Agraria La Molina. Programa Doctorado Agricultura Sustentable. Facultad de Agronomía. e-mail: ajo@lamolina.edu.pe

${ }^{3}$ Universidad Nacional Agraria La Molina. Programa Doctorado Agricultura Sustentable. Facultad de Agronomía. e-mail: mcanto@lamolina.edu.pe.

${ }^{4}$ Universidad Nacional Agraria La Molina. Programa Doctorado Agricultura Sustentable. Facultad de Agronomía. e-mail: husovi@lamolina.edu.pe

${ }^{5}$ Universidad Nacional Agraria La Molina. Programa Doctorado Agricultura Sustentable. Facultad de Agronomía. e-mail: svargas@lamolina.edu.pe

${ }^{6}$ Consejo Nacional de Ciencia, Tecnología e Innovación Tecnológica - CONCYTEC. e-mail: phuerta@concytec.gob.pe
} 